\title{
Mirror Assembly Controller Based on S7-200 PLC
}

\author{
Qiang-qiang RONG ${ }^{1}$ Jin-hua DING ${ }^{1}$ Xiao-jing LEI $^{1}$ Yu-jun $\mathrm{HU}^{1}$ \\ Wei-fu WANG ${ }^{1}$ Wen-jing LI ${ }^{1}$ \\ ${ }^{1}$ Dalian Polytechnic University, Dalian, China
}

\begin{abstract}
Aimed at features of mirror assembly process, a mirror assembly controller with servo motor as actuators was developed, based on S7-200 PLC and position control module EM253. According to the working principle of mirror assembly, the mirror positioning, mirror frame orientation and manipulator working process were described. This control system realized precise position control of the manipulator and the location error could be reached $30 \mu \mathrm{m}$. The hardware and software of the mirror assembly controller was also discussed.
\end{abstract}

Keywords: Mirror assembly; S7-200 PLC; Servo motor; EM253

\section{Introduction}

Mirror assembly is one of the processes in furniture production line. This process involves mirror frame orientation, mirror positioning, mirror suction, mirror moving, blowing, etc. The key technology is how to ensure positioning accuracy of the picture frame and the manipulator. Position precision of the manipulator mainly depends on the motion controller and the servo driver. High-end control module can realize complicated motion, but the cost is high and the structure is complicated ${ }^{[1]}$.

Mirror assembly process need accurate position control, but its trajectory is not complex, so there is no need to choose the expensive high-end motion control system. Therefore a simple and reliable servo control system is developed, using Siemens S7-200 programmable controller and EM253 position control module, realizing control of the high precision motion of the manipulator.

\section{Structure of the Mirror Assembly System}

As figure 1 shows, mirror assembly system is composed of three parts: mirror positioning device, mirror frame positioning device and mirror moving manipulator device.

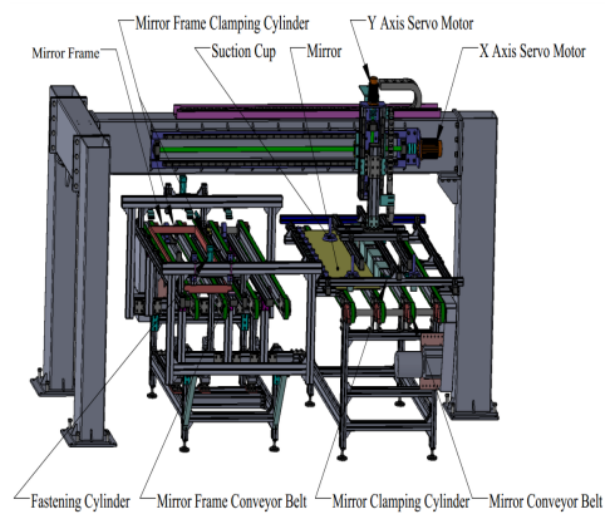

Fig. 1: Structure of the mirror automatic assembly system.

Mirror position fixing device includes mirror conveyor belt, mirror locating piece and clamping cylinder, as shown in 
figure 1. Mirror is conveyed by the conveyor belt, it should be stopped when meeting locking block 1 . At this moment, there is a deviation $\delta_{1}$ (as shown in figure 3 ) between mirror and locking block. When clamping cylinder complete its firstly positioning, the clearance $\delta_{1}$ is eliminated, but a new deviation $\delta_{2}$ (as shown in figure 4) will be produced. Restart belt motor again (the running time is 1s). After motor stops, the clamping cylinder should make its secondary positioning, then the mirror is absolutely located. Structure of the mirror position fixing devices is shown in figure 2 .

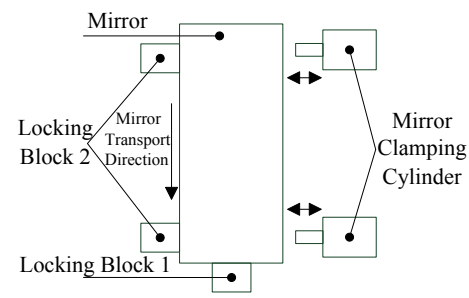

Fig. 2: Structure of the mirror position fixing device.

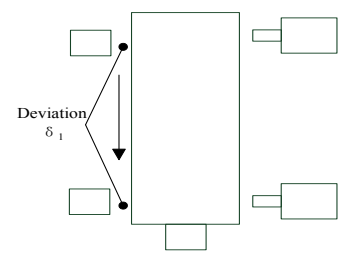

Fig. 3: Deviation $\delta_{1}$ of the mirror position fixing.

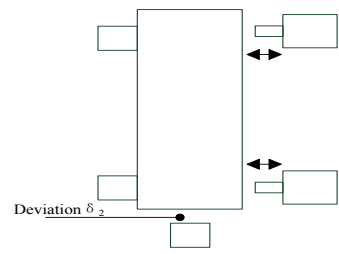

Fig. 4: Deviation $\delta_{2}$ of the mirror position fixing.

Position fixing device of the mirror frame is made up by mirror frame delivery motor, clamping cylinders, fastening cylinder and locating piece. Position fixing principle of the frame is the same as the mirror's. After mirror frame is fully positioned, fastening cylinder will press the frame and make it in its right position.

Mirror moving manipulator device is consist of $\mathrm{X}$ axis servo motor, $\mathrm{Y}$ axis servo motor (brake motor), suction cup, screws, guide rails, etc. Servo motor controls position of the suction cups, realizing operations as glass suction, moving, holding and releasing. The size of the mirror frame is $400 \mathrm{~mm} \times 1600 \mathrm{~mm}$ and the mirror size is $398 \mathrm{~mm} \times 1598 \mathrm{~mm}$, so the maximum allowable assembly deviation is $0.5 \mathrm{~mm}$ at each side, which puts forward a strict requirement of the manipulator precision.

\section{Hardware Design of the Control System}

\subsection{Hardware Configuration of the System}

Mirror assembly control system consists of PC (touch screen), lower machine (Siemens S7-200 series PLC CPU226), position control module (EM253), ac servo motor and servo driver (ADSMS110-060M-ac servo motor), encoder, etc. Figure 5 shows principle structure of the control system.

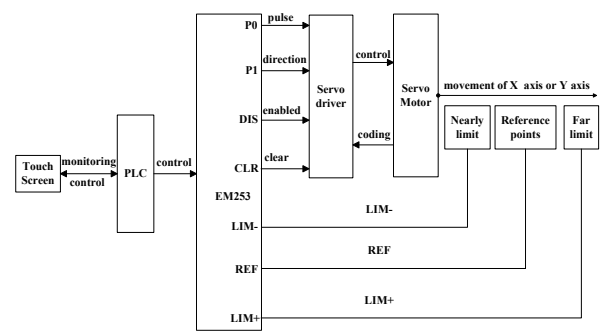

Fig. 5: Principle structure of the control system. 
Upper computer uses Siemens smart1000 touch screen, and it makes communication with PLC through serial port (RS485). Upper machine sets movement parameters, realizing position control of the manipulator, monitoring working condition of the executive parts, variable register, etc.

\subsection{Position Control Module EM253}

Position control module EM253 controls position and speed of the moving manipulator. It is a special function module of S7-200 PLC. It communicates with PLC through I/O port bus, producing pulse for servo motor ${ }^{[2]}$. Figure 6 shows the connection way between EM253 module and servo motor driver. EM253 belongs to open loop control mode, so it can't feedback real state of the manipulator well ${ }^{[3]}$. Using servo driver's differential output signal and the encoder feedback signal, closed loop control can be realized by setting proper parameters of the servo driver.

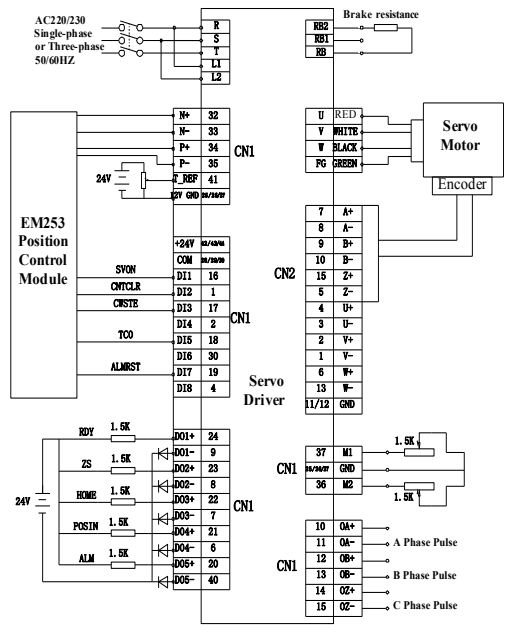

Fig. 6: The connection way between EM253 module and servo motor driver.

\section{Software Design of the System}

\subsection{Main program design}

Control system uses point control mode and modular design thought. PLC control program is divided into four parts:

- system startup subroutine

- initialization subroutine

- servo motor subroutine

- mirror frame orientation and mirror positioning subroutine

\subsection{Functions of the Subroutine}

System startup subroutine controls state of the whole system through gathering external start and stop signal, using the typical start, keep and stop control circuit to achieve.

Initialization subroutine triggers the register SM0.1 or SM0.0 to achieve servo motor initialization operation ${ }^{[4-5]}$, establishes system zero point coordinates, assigns an initial value for registers through the MOV instruction.

Servo motor subroutine controls EM253 module to output a certain number of pulse and frequency ${ }^{[6]}$, using commands as POSx_GOTO and POSx_RUN, realizing single speed positioning operation of the manipulator. Figure 7 introduces software process of the servo motor subroutine.

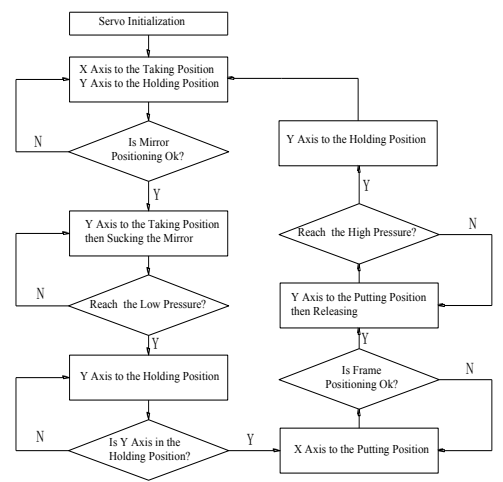

Fig. 7: Software process of the servo motor subroutine. 
Mirror frame orientation and mirror positioning subroutine completes transport and positioning work of the picture frame and mirror through controlling belt machine and the action of the related cylinder

\section{Results}

Graph of the mirror moving manipulator position error is shown in figure 8 . After pilot production of the system, it has been formally used in production. Through statistics and analysis maximum position error of the manipulator in a particular situation that the manipulator executives 1000 times strokemm in full load cases and respectively in different speed, we could draw a conclusion that this system can realize precise localization of the manipulator. Actual measurement shows that the $\mathrm{X}$ axis biggest position error is $30 \mu \mathrm{m}$ and the $\mathrm{Y}$ axis biggest position error is $28 \mu \mathrm{m}$. It can meet the mirror assembly accuracy.
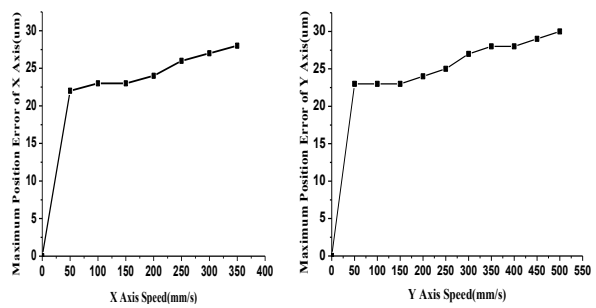

Fig. 8: Graph of the maximum position error of the Manipulator

\section{Conclusion}

Using Siemens S7-200 PLC and position control module EM253, making full use of advantages of the control module in high precision motion control aspects, the mirror automatic assembly control system is developed. The circuit composition is simple and convenient, and it is easy to install and debug. The system can realize accurate position control and it can be applied to other position control occasions, to achieve production automation.

\section{References}

[1] Hu Jiali, Yan Baorui, Zhang Anzhen. Application of S7-200 PLC in Position Control of Servo Motor[J]. Process Automation Instrumentation, pp. 38-40, 2009(30).

[2] Xu Lumei, Zheng Guohjua, Li Ping. The Design of Welding Torch Swinging Control System Based on CPU224XP $[\mathrm{J}]$. Applied Energy Technology, pp. 45-47, 2009(1).

[3] Lin Song. Application of the Siemens Control Module in Assembly System[J]. Science \& Technology Information, pp. 324-325, 2006(9).

[4] Gao Anbang, Tian Min, Yu Ning. Siemens S7-200 PLC Application Design[M]. Bei Jing: China Machine Press, pp. 247-250, 2011.

[5] S7-200 PLC User Manual. 2004.

[6] Tao Xian, Yang Zesheng. PLCbasede Control System X-Y Table Design[J]. Development \& Innovation of Machinery \& Electrical Products, pp. 131-132, 2012(25). 